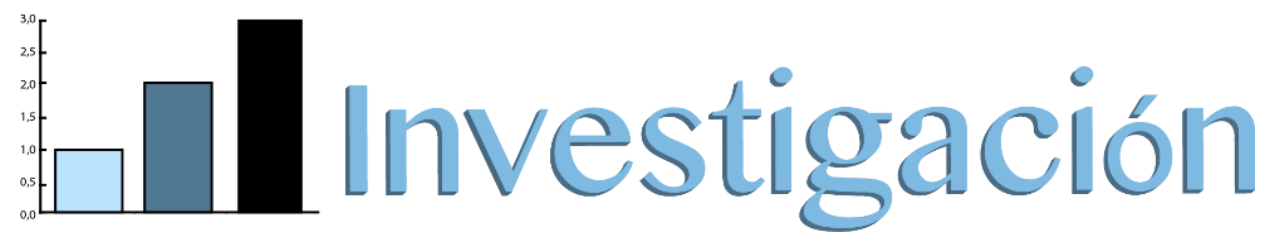

\title{
RELACIÓN ENTRE COMPORTAMIIENTOS DE RIESGO EN SEXUALIDAD Y FAMILIA EN ADOLESCENTES ESCOLARIZADOS, 2015-2016.
}

\section{Relationship between sexuality risk behaviors and family in school enrolled adolescents during 2015-2016.}

Luisa Fernanda Molano, Natalia Carolina Montes, Daniela Alejandra Moreno, Laura Camila Nova, Erika Daniela Olaya, Jenny Carolina Páez ${ }^{\ddagger}$ Tutor: Juan Carlos González§ Manejo estadístico y edición: Jenny Pinzón ${ }^{* *}$ Dibujos: Andrés Reyes ${ }^{\dagger+}$

\section{Resumen}

Objetivo: Estimar si la estructura y la percepción de la funcionalidad familiar están relacionadas con comportamientos de riesgo de adolescentes escolarizados en torno a la sexualidad.

Metodología: Estudio observacional de corte transversal, realizado en 3217 adolescentes con edades comprendidas entre los 10 y los 20 años, que asistían a cuatro colegios públicos de la localidad de Suba durante los años 2015 y 2016. Se aplicó una encuesta autodiligenciada, anónima y voluntaria, de 62 preguntas a través de las cuales se indagó por riesgos psicosociales. Para el presente estudio se tomaron las variables relacionadas con comportamientos sexuales de riesgo (ha tenido relaciones sexuales y si planificó en la primera relación) y se compararon con el género, la estructura y la percepción de la funcionalidad familiar.

Manejo estadístico: La información recolectada de los cuatro colegios se tabuló en una base de datos elaborada con el programa EXCEL. Las preguntas de comportamientos de riesgo fueron analizadas con el programa EPIINFO obteniendo las razones de riesgo, con sus intervalos de confianza y valores de prueba $p$.

Resultados: Edad media de la población, 15 años. Prevalencia de relaciones sexuales, $25 \%$; edad promedio de inicio, 14,1 años. Disfunción familiar severa en el $5 \%$ de los hogares y el $32 \%$ son nucleares incompletos. Las mujeres tienen menos relaciones sexuales que los hombres (OR 1,9; IC $95 \% 1,6-2,2$ ). Cuando el adolescente percibe de manera disfuncional a su familia, hay mayor riesgo de inicio de vida sexual (OR 2,1; IC $95 \% 1,5-2,9)$.

Conclusión: La percepción de la funcionalidad familiar es un importante factor de protección contra los riegos en los comportamientos sexuales de los jóvenes.

Palabras claves: Adolescente, conducta del adolescente, sexualidad, conducta sexual, relaciones familiares, anticoncepción.

\begin{abstract}
Objective: To assess the relationship between structure and perception of family functionality and risk behaviors in sexuality of school enrolled adolescents.

Methodology: Gross-sectional study carried out on 3217 adolescents whose ages ranged from 10 to 20 years and who were attending four public schools from Suba locality, during 2015 and 2016. A voluntary self-questionnaire of 62 questions about psychosocial risks was administered. The variables related to sexual behavior (to have intercourse and to use contraception method in the first sexual relation) were selected. Then those variables were compared with gender, structure and family functionality.

Statistical management:Theinformation collected was tabulated in a database created in Excel. Questions about risk behavior were analyzed by Epi Info in order to obtain the odds ratio, confidence intervals and $p$ value.

Results: Average age is 15 years. Sexual relations prevalence, $25 \%$ and the onset average age was 14,1 years. Severe dysfunction was found in $5 \%$ of households and $32 \%$ of them were incomplete nuclear. Females reported less sexual activity than males (OR 1,$9 ; 95 \%$ CI 1,6-2,2). Adolescent have more risk of intercourse when they perceive family dysfunction (OR 2,1; $95 \%$ CI 1,5-2,9). Conclusion: To perceive a good family function is an important protection factor against young's sexual behavior risks.
\end{abstract}

Key words: Adolescent, adolescent behavior, sexuality, sexual behavior, family relations, contraception.

* Estudiantes VII semestre - Facultad de Medicina FUJNC.

$\S$ MD. Especialista Medicina Familiar Integral, MSP. Director Departamento de Medicina Comunitaria - juan.gonzalez@juanncorpas.edu.co

** Coeditora Carta Comunitaria - jenny.pinzon@juanncorpas.edu.co

${ }^{+\dagger}$ MD Cirujano General. 


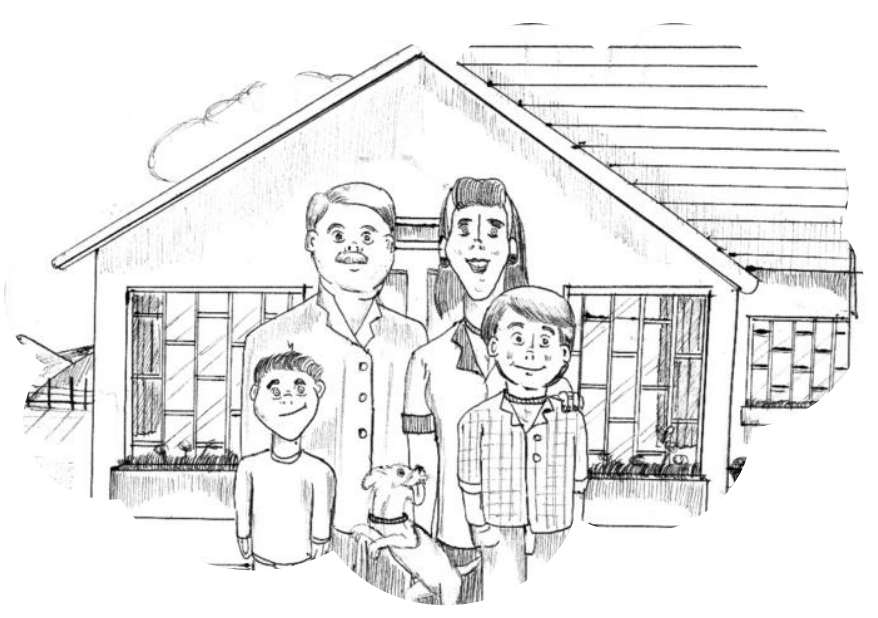

Como lo expresan Mendizábal et al, "la vida en familia proporciona el medio para la crianza de los hijos, es la influencia más temprana y duradera para el proceso de socialización y, en buena medida, determina las respuestas de sus integrantes entre sí y hacia la sociedad. La estructura de una familia se determina por las pautas de interacción que se establecen entre sus miembros, quienes se relacionan dentro de un sistema de manera altamente recíproca, reiterativa y dinámica" (1). Así, el núcleo familiar con buena funcionalidad, que se caracteriza por sus relaciones de intimidad, solidaridad y por ser una red de apoyo, constituye un ejemplo y un rol permanente de cada integrante, convirtiéndose en un agente estabilizador que se manifiesta en las funciones sociales de crianza, desarrollo emocional, comunicación y transmisión de pautas conductuales y valorativas (2).
Gómez et al (3) afirman que una familia percibida como disfuncional por parte del hijo adolescente es considerada como factor de riesgo para el desarrollo de conductas poco saludables y disminución del bienestar emocional (3).

La etapa adolescente supone un proceso continuo de maduración, independencia y búsqueda de identidad; todo esto trae consigo inquietudes con respecto a la sexualidad y, en la mayoría de las ocasiones, los jóvenes resultan vulnerables y expuestos a la obtención de información muy variada, ya sea acertada, errónea o simplemente innecesaria, dependiendo de la fuente (relatos de pares, clases de maestros o información brindada por padres, siendo la última la referencia más importante). Al respecto, Domínguez (4) afirma que "la relación familia-adolescencia debe soportarse bajo una comunicación fluida en torno a la sexualidad, lo cual marca el modelo formador de actitudes y acciones saludables en este plano de la vida".

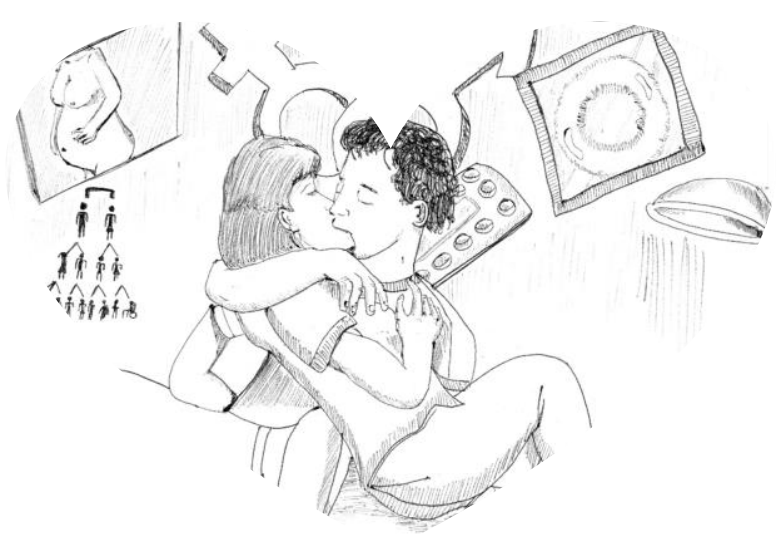

Dentro del marco de la atención primaria en salud son esenciales las medidas de prevención de enfermedades de transmisión sexual y embarazos no deseados en población adolescente, para lo cual deben detectarse factores de riesgo y factores protectores tales como la anticoncepción, la enseñanza de valores ético morales y el importante rol de la familia (4).

Es claro entonces que la funcionalidad familiar es clave para el control de conductas de riesgo en la vida sexual de los adolescentes; son los padres quienes deben tomar el mando y abordar el tema con los jóvenes, teniendo a su disposición información clara y amplia, analizando cada aspecto tranquila y respetuosamente. Es importante que los padres no omitan este tipo de comunicación.

Carvajal et al (5) señalan que "muchos adultos piensan que ofrecer información sobre sexualidad a los adolescentes es motivo de preocupación, ya que consideran que los jóvenes son sexualmente promiscuos y que, suministrándoles nueva información, aumentaría esa promiscuidad", así que, omiten este proceso educativo y el adolescente resulta desinformado y en riesgo aun sin pertenecer a una familia disfuncional. 


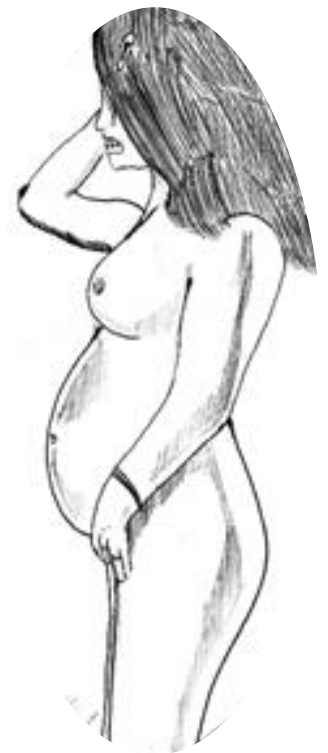

Según el Boletín 2 "El Aumento del Embarazo de adolescentes en Colombia" (6), que expone, entre otros datos, la edad de inicio de la vida sexual en jóvenes de 15 a 25 años de edad, se logra concluir que cada vez la actividad sexual tiende a comenzar en edades mucho más tempranas (una de cada dos personas en la adolescencia) y con mayor frecuencia en jóvenes con bajos niveles de educación e ingresos, así como en habitantes de áreas rurales. Asimismo, el boletín de prensa del DANE 2013, sobre la "Encuesta de conocimientos y actitudes en torno a la sexualidad en niños, niñas y adolescentes escolarizados" (7), realizada bajo el mandato de la ley 1336 de 2009, señala que en ciudades como Bucaramanga y Manizales la edad de inicio de la vida sexual está en alrededor de los 15 a los 17 años (con mayor incidencia en la ciudad de Manizales).

Es claro entonces que durante la etapa adolescente se requiere de un mayor acompañamiento y de orientación sobre las conductas de riesgo relacionadas con la sexualidad y que los actores responsables de dicho acompañamiento son quienes más se acercan a su cotidianidad, es decir, el colegio, pero, sobre todo, la familia (3).

Por otro lado, con relación al género, Vega (8) indica que "los hombres que inician antes las relaciones sexuales, tienen más parejas ocasionales y corren mayor riesgo que la mujeres. En cambio, las chicas suelen tener relaciones sexuales en el marco de una relación estable y asociada a una relación de amor y confianza". Estas diferencias en el comportamiento sexual que se atribuyen al género se denominan 'diferencias de género'. Adicionalmente, el autor manifiesta que, "respecto del género, los tipificados como masculinos o andróginos (los/as adolescentes que se definen como andróginos o masculino) son más erotofílicos y los femeninos e indiferenciados son más erotofóbicos (8).

En concordancia con lo anterior, la Encuesta del DANE (7), mostró que el $57,5 \%$ de los niños y el $42,5 \%$ de las niñas encuestados informaron haber tenido relaciones sexuales sin ningún tipo de protección o método anticonceptivo y la razón principal encontrada en los resultados fue, que "cuando se dio el momento no los tenía a disposición"; otras causas encontradas para la evasión del uso del condón como método anticonceptivo son la falta de dinero, no querer utilizarlo, el no estar disponible, la vergüenza de conseguirlo y la desinformación (9).

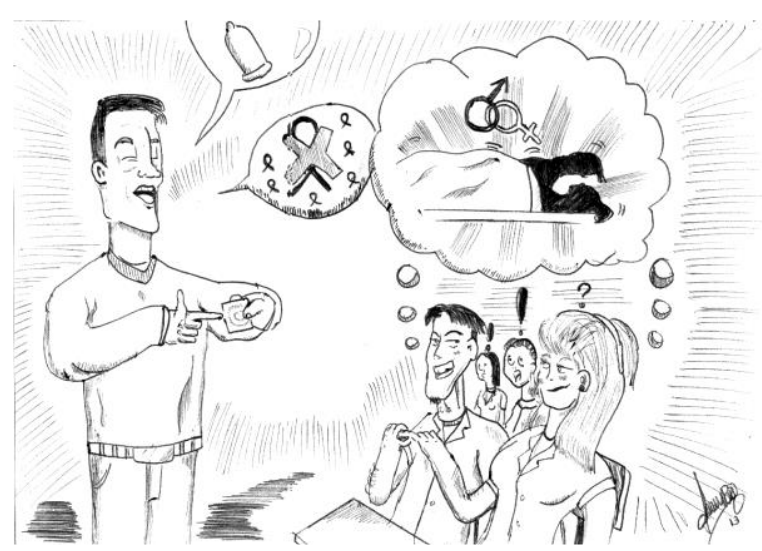

El riesgo de no utilizar métodos anticonceptivos en la primera relación sexual no solamente se relaciona con la alta probabilidad de embarazo no deseado, sino también con el riesgo de adquirir enfermedades de transmisión sexual. La comunicación familiar y la información que se proporcione sobre el tema resultan claves en la prevención de dichos eventos al promover el uso de anticoncepción tanto en la primera relación sexual como en las posteriores. Como lo expresan Lavielle et al, "los adolescentes que perciben una comunicación abierta tienden a conductas de sexo seguro, entre ellas el inicio de la vida sexual a una edad no temprana, tener pocas parejas sexuales y el uso frecuente del condón" (9).

La encuesta del DANE antes mencionada (7) encontró que el $2,2 \%$ de los encuestados afirmó haber estado en embarazo o haber dejado en embarazo a alguna mujer (7), lo cual reafirma que los jóvenes son mucho más vulnerables al inicio de vida sexual con comportamientos de riesgo tales como el desconocimiento de la utilidad de métodos anticonceptivos o la evasión de su uso (7).

Es importante resaltar que los adolescentes no están haciendo uso de los métodos anticonceptivos, siendo estos una prevención importante de enfermedades y embarazos y que el ambiente familiar es una fuente primordial para la educación temprana acerca del tema. Dada la trascendencia de estos temas, la presente investigación indagó sobre la relación que existe entre los comportamientos de riesgo en torno a la sexualidad en adolescentes y la funcionalidad y estructura de la familia, con el fin de contribuir a la promoción de programas dirigidos a los padres de los colegios asociados, que los alerten sobre la necesidad de su compromiso con la vida sexual de sus hijos adolescentes y así disminuir el riesgo de efectos adversos, entre ellos el embarazo no deseado y las enfermedades de transmisión sexual. 


\section{MATERIALES Y MÉTODOS}

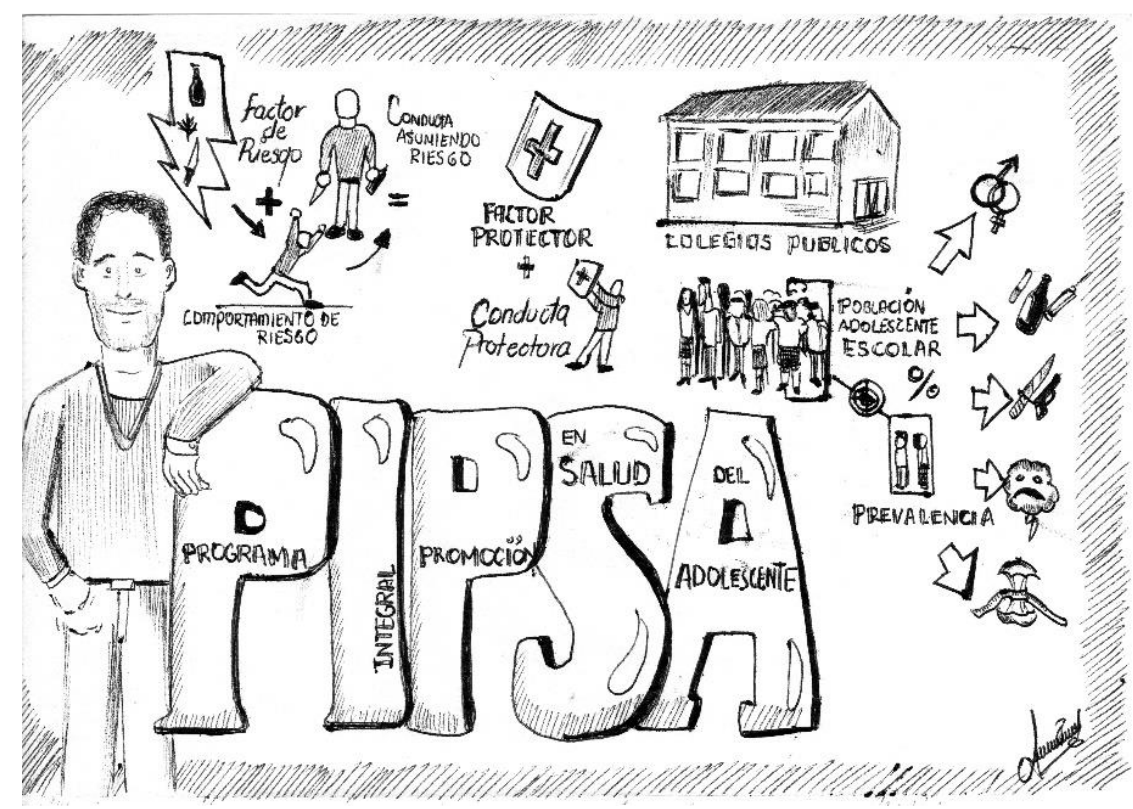

Tipo de estudio: estudio observacional de corte transversal en adolescentes escolarizados de la localidad de Suba (Bogotá).

Población de estudio: estudiantes de bachillerato con edades comprendidas entre los 10 y los 20 años que asistían a cuatro instituciones educativas distritales de la localidad de Suba pertenecientes al proyecto PIPSA (Programa Integral de Promoción de Salud del Adolescente).

Criterios de inclusión: Esta investigación forma parte del proyecto PIPSA que se viene adelantando desde 2005 en la localidad de Suba por parte de la facultad de Medicina de la Fundación Universitaria Juan N. Corpas. Los criterios de inclusión fueron pertenecer a alguno de los cuatro colegios seleccionados, estar cursando algún grado de bachillerato $\left(6^{\circ}\right.$ a $\left.11^{\circ}\right)$, presentar previo consentimiento informado firmado por los padres y querer participar en el estudio comprometiéndose a hacerlo con seriedad y total sinceridad. Se accedió a la población para la realización de un cuestionario a través de permisos concedidos por las directivas. A todos se les informó que la realización de la encuesta sería de manera anónima y voluntaria.

Variables de estudio: Se seleccionaron como variables independientes las características demográficas de edad, género y estratos socioeconómicos (identificados en una escala de 1 a 6 , expresando los más bajos un mayor nivel de pobreza); variables referentes a la estructura familiar evaluando la presencia de familias nucleares completas (padre, madre e hijos) o incompletas (ausencia de uno de los padres), reconstituidas (con padrastro/a) y, la funcionalidad familiar, usando el test de APGAR familiar que mide a través de cinco preguntas la percepción hacia la ayuda que siente por parte de la familia para resolver problemas, participar en decisiones, el afecto y los espacios para compartir, pudiendo en cada pregunta contestar "casi siempre" ( 2 puntos), "a veces" (1 punto) y "casi nunca" (0 puntos). De acuerdo al puntaje obtenido en el test, la funcionalidad familiar se clasifica como buena (de 8 a 10 puntos), disfunción moderada (de 4 a 7 puntos) y disfunción severa (de 0 a 3 puntos). Las variables dependientes corresponden a las preguntas sobre comportamientos de riesgo en torno a la sexualidad (tener o haber tenido relaciones sexuales y protección durante la primera relación sexual, para ambas preguntas las opciones de respuesta fueron "Sí" o "No").

Recolección de la información: Las encuestas se llevaron a cabo durante los períodos escolares de 2015 y 2016. La realización de cada cuestionario autodiligenciado tomó en promedio 20 minutos. A los colegios acudieron estudiantes de Medicina, previo consentimiento informado firmado por los padres de los estudiantes, para la explicación y aplicación de las encuestas. Al llegar a los salones de clase, se les informó a los jóvenes que la encuesta debería ser contestada con la mayor sinceridad y seriedad posibles y que, por eso, sería voluntaria y podían preguntar sobre cualquier cosa que no se entendiera del cuestionario. Se insistió en que los estudiantes tendrían el derecho a no participar y que sus respuestas se mantendrían bajo total anonimato; además, los docentes no estuvieron presentes durante la actividad. Posterior al análisis de los resultados, cada colegio participante recibió un informe escrito con el diagnóstico de riesgos.

Manejo estadístico: La información recolectada de los cuatro colegios fue tabulada en una base de datos diseñada con el programa EXCEL. La relación entre las variables independientes de interés para el estudio (género, estructura y funcionalidad familiar) y las preguntas de comportamientos de riesgo (haber tenido relaciones sexuales $y$ haber planificado en la primera relación) fue analizada con el programa EPIINFO a través del cual se obtuvieron las razones de riesgo, los intervalos de confianza y los valores de la prueba $p$. Dado que algunos estudiantes no respondieron todas las preguntas de la encuesta, el porcentaje de estas se calculó con el total contestado respectivamente. 


\section{RESULTADOS}

La edad promedio es de 15 años, la moda está ubicada en el rango de los 13 a los 16 años y la mayoría de la población es de estratos 3 y 4 seguidos de los de estratos 1 y 2 .

Tabla 1. Características sociodemográficas de adolescentes escolarizados de la localidad de Suba (Bogotá) en 2015 y 2016.

\begin{tabular}{lrr}
\hline $\begin{array}{c}\text { CARACTERÍSTICAS DE LA } \\
\text { POBLACIÓN }\end{array}$ & \multicolumn{1}{l}{ No $^{\circ}$} & \% \\
\hline Edades & 710 & $\mathbf{2 2}$ \\
De 10 a 12 años & 2155 & $\mathbf{6 7}$ \\
De 13 a 16 años & 332 & $\mathbf{1 0}$ \\
De 17 a 20 años & & \\
Sexo & 1603 & $\mathbf{5 0}$ \\
Masculino & 1614 & $\mathbf{5 0}$ \\
Femenino & & \\
Estrato & 1487 & $\mathbf{4 9}$ \\
Estratos 1 y 2 & 1505 & $\mathbf{5 0}$ \\
Estratos 3 y 4 & 38 & $\mathbf{1}$ \\
Estratos 5 y 6 & & \\
\hline
\end{tabular}

Tabla 2. Características de estructura, función familiar y riesgo de comportamientos en sexualidad en población adolescente escolarizada de la localidad de Suba

(Bogotá), 2015 y 2016.

\begin{tabular}{lrr}
\hline \multicolumn{1}{c}{ VARIABLE } & No & \% \\
\hline Estructura familiar & & \\
Hogar nuclear (vive con papá, mamá y hermanos). & 1649 & $\mathbf{5 3}$ \\
Hogar incompleto (falta uno de los padres). & 1007 & $\mathbf{3 2}$ \\
Hogar extenso (padres, hermanos y otros). & 260 & $\mathbf{8}$ \\
Hogar reconstituido (padrastro y/o madrastra). & 73 & $\mathbf{2}$ \\
Hogar sin padres. & 138 & $\mathbf{4}$ \\
Funcionalidad familiar & 147 & $\mathbf{5}$ \\
Disfunción severa. & 424 & $\mathbf{1 4}$ \\
Disfunción moderada. & 2488 & $\mathbf{8 1}$ \\
Funcionalidad normal. & & \\
Riesgos de sexualidad & 791 & 25 \\
Tener o haber tenido relaciones sexuales. & 474 & 16 \\
\hline Protección en primera relación sexual. & & \\
\hline
\end{tabular}

La buena función familiar ( $81 \%$ ) junto con el hogar nuclear (53 \%) constituyen las mayores prevalencias. Uno de cuatro jóvenes de bachillerato ya inició vida sexual. 
Tabla 3. Razones de riesgo por género, estructura y funcionalidad familiar versus comportamientos de riesgo en sexualidad en población adolescente escolarizada de la localidad de Suba (Bogotá), 2015 y 2016.

\begin{tabular}{|c|c|c|c|c|c|c|c|c|}
\hline VARIABLE & \multicolumn{2}{|c|}{ Sí } & \multicolumn{2}{|c|}{ No } & OR & LI & LS & Prueba P \\
\hline \multicolumn{9}{|c|}{ Ha tenido relaciones sexuales } \\
\hline Nuclear incompleta & 263 & 26,9 & 716 & 73,1 & \multirow{2}{*}{1,2} & \multirow{2}{*}{1} & \multirow{2}{*}{1,4} & \multirow{2}{*}{0,0602227} \\
\hline Nuclear completa & 381 & 23,6 & 1235 & 76,4 & & & & \\
\hline \multicolumn{9}{|c|}{$\begin{array}{l}\text { Usó algún método de planificación } \\
\text { en su primera relación }\end{array}$} \\
\hline Nuclear incompleta & 167 & 60,1 & 111 & 39,9 & \multirow{2}{*}{0,8} & \multirow{2}{*}{0,6} & \multirow{2}{*}{1,1} & \multirow{2}{*}{0,1876155} \\
\hline Nuclear completa & 230 & 55 & 188 & 45 & & & & \\
\hline \multicolumn{9}{|c|}{ Ha tenido relaciones sexuales } \\
\hline Disfunción severa & 57 & 39 & 89 & 61 & \multirow{2}{*}{2,1} & \multirow{2}{*}{1,5} & \multirow{2}{*}{2,9} & \multirow{2}{*}{0,0000328} \\
\hline Buena función & 580 & 23,8 & 1858 & 76,2 & & & & \\
\hline \multicolumn{9}{|c|}{$\begin{array}{l}\text { Usó algún método de planificación } \\
\text { en su primera relación }\end{array}$} \\
\hline Disfunción severa & 33 & 54,1 & 28 & 45,9 & \multirow{2}{*}{1,2} & \multirow{2}{*}{0,7} & \multirow{2}{*}{2} & \multirow{2}{*}{0,5896513} \\
\hline Buena función & 353 & 57,7 & 259 & 42,3 & & & & \\
\hline \multicolumn{9}{|c|}{ Ha tenido relaciones sexuales } \\
\hline Hombre & 484 & 31,1 & 1075 & 69 & \multirow{2}{*}{1,9} & \multirow{2}{*}{1,6} & \multirow{2}{*}{2,2} & \multirow{2}{*}{0,0000000} \\
\hline Mujer & 305 & 19,3 & 1278 & 80,7 & & & & \\
\hline \multicolumn{9}{|c|}{$\begin{array}{l}\text { Usó algún método de planificación } \\
\text { en su primera relación }\end{array}$} \\
\hline Hombre & 287 & 54,3 & 242 & 45,8 & \multirow{2}{*}{1,2} & \multirow{2}{*}{0,9} & \multirow{2}{*}{1,6} & \multirow{2}{*}{0,1628616} \\
\hline Mujer & 187 & 59,2 & 129 & 40,8 & & & & \\
\hline
\end{tabular}

La disfunción familiar y el género establecieron relaciones significativas con tener o haber tenido relaciones sexuales.

\section{DISCUSIÓN}

Este tipo de investigaciones favorece el sesgo que puede presentarse cuando algunos de los participantes no diligencian la encuesta con la sinceridad necesaria. Para enfrentarlo se insistió en la voluntariedad y anonimato de la encuesta.

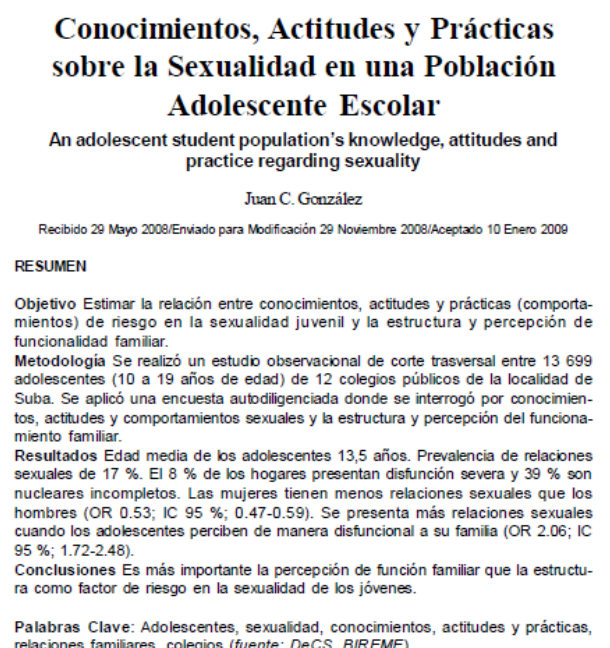

Palabras Clave: Adolescentes, sexualidad, conocimien 
Al comparar las características familiares (ver tabla 2) con el estudio realizado por González (10) en el año 2006, sobre conocimientos, actitudes y prácticas en torno a la sexualidad en adolescentes escolarizados, en población similar de la misma localidad y en los mismos estratos (10), se detectó que la cantidad de hogares nucleares va en descenso (59\% en 2006) lo que puede sugerir una nueva dinámica de la estructura familiar.

Se observa una mejoría en la función familiar medida a través del APGAR familiar (buena función $81 \%$ en este estudio frente a $68 \%$ en 2006), tal vez como consecuencia de una mayor preparación hacia la convivencia en los mismos colegios.

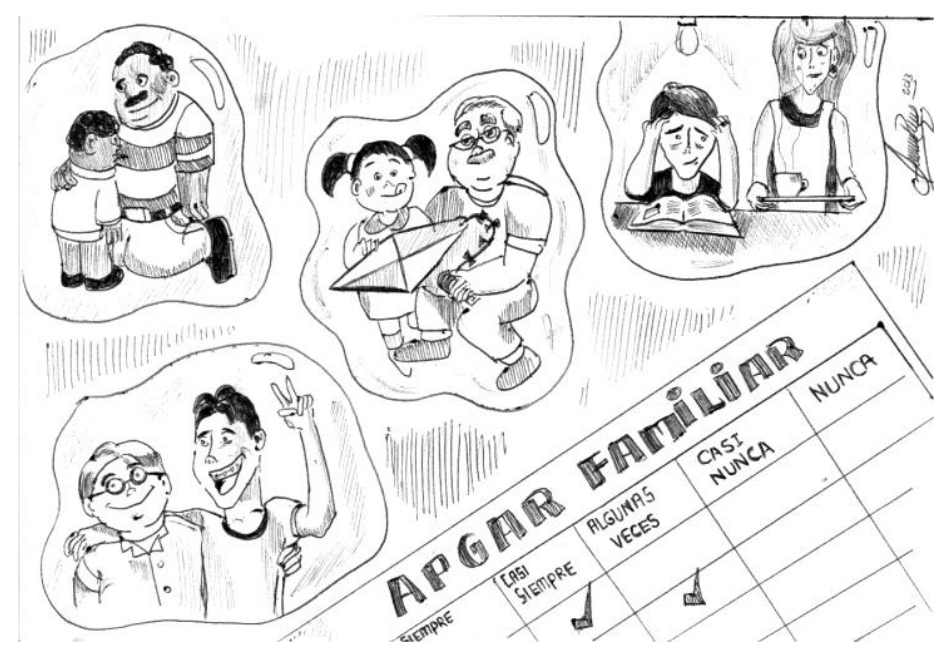

González (10) en su estudio confirma la funcionalidad familiar como un factor protector sobre las conductas de riesgo sexual en adolescentes, lo que es coherente con esta investigación que muestra que, a mayor disfunción, más relaciones sexuales (tabla 3 ), quizá eso se deba a que existe menos comunicación familiar. Este hallazgo lo confirman Salazar et al (11), quienes afirman que "Ios adolescentes que tienen mala o regular comunicación con sus padres tienen grados de conocimiento malos o muy malos sobre sexualidad, así como mayor probabilidad de inicio de vida sexual a edades tempranas $y$, en muchas ocasiones, sin protección", exponiéndose de esa forma a más desinformación y a los riesgos y eventos desfavorables derivados de esa situación tales como embarazo adolescente, morbimortalidad materna, infecciones de transmisión sexual, abortos e, incluso, abandono de escolaridad; situaciones que a la larga van a terminar empeorando aún más la condición familiar. Asimismo, Santander et al (12) destacan en su estudio la importancia de la influencia familiar como factor protector de conductas de riesgo en jóvenes y concluyen que "el riesgo de haber tenido relaciones sexuales fue significativamente mayor en adolescentes de hogares percibidos como disfuncionales en comparación a hogares percibidos como funcionales" (12).

Santander et al (12) manifiestan que los adolescentes de familias con disfuncionalidad perciben que "sus padres no les dedican el tiempo adecuado para conversar, además, refieren falta de conocimiento de las actividades de sus hijos, falta de reglas claras en la casa, ausencia de rituales familiares y de actividades conjuntas. Todo lo cual podría estar relacionado con el nivel sociocultural de las familias, que impide una conexión adecuada con los hijos, lo que, sumado a distorsiones en las representaciones mentales acerca de la tarea educativa del padre y la madre, determinaría la presencia de mayores conductas de riesgo en escolares". Además refiere (12) que "la disponibilidad de tiempo de los padres hacia sus hijos, la presencia de rituales familiares y las actividades compartidas son factores protectores. La presencia de un adecuado vínculo familiar disminuye significativamente la posibilidad de todas las conductas de riesgo en el adolescente, a pesar de la existencia, en el mismo adolescente, de factores de riesgo significativos".

No se encontraron relaciones significativas entre la estructura familiar con los conocimientos, actitudes y prácticas indagados. Pérez (13) respalda estos resultados ya que también refiere encontrar que la estructura familiar no influye en la manera en que el adolescente aprecia y percibe el mundo, siendo la funcionalidad lo importante (13). Este hecho es coincidente con lo encontrado por González en su estudio de 2006 (10).

En cuanto al género, en la tabla 3 muestra que, en este estudio, son los hombres quienes tienden a tener más vida sexual que las mujeres e iniciarla más temprano (en una relación $2: 1$ ). Una posible explicación que se podría tener al respecto, la encontramos en Márquez (14), quien refiere que "la actividad sexual, tiene distintos significados para hombres y mujeres". Mientras las chicas depositan en el sentimiento su decisión de tener relaciones con la idea de una pareja estable y duradera, los hombres las conciben como parte de su experiencia de "conocer el mundo". Es de suponerse que tales significados conllevan puntos de vista distintos frente a un mismo hecho; por un lado el romanticismo, cultivar y vivir el sentimiento para sí mismo $y$, por el otro, la experimentación, reforzar su hombría y habilidad para mostrarse ante los demás, en el caso de los varones. De ahí que la sexualidad se viva de manera distinta, como lo indican los resultados del presente estudio; las mujeres, en su mayoría, sostienen relaciones con sus novios o parejas permanentes y los hombres reportan más encuentros ocasionales repetitivos. 


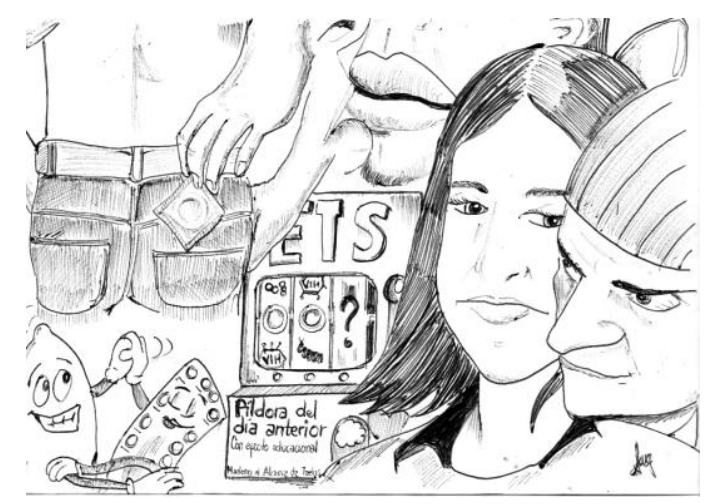

Tampoco se detectó en el presente estudio que haya relación estadística significativa entre usar métodos anticonceptivos en la primera relación sexual y la estructura y funcionalidad familiar ni con el género (tabla 3). Obviamente, aunque los adolescentes sean informados acerca de la importancia de utilizarlos, son ellos los responsables de darles un adecuado y oportuno uso y con ello evitar embarazos no deseados así como ETS. Este hallazgo es interesante e indica que conviene hacer mucho más énfasis en educar sobre su uso, no solo a nivel familiar sino escolar. Lavielle (9), en su estudio sobre el impacto de la familia en las conductas sexuales de riesgo de los adolescentes, hizo una interesante lista de las razones para no utilizar condón expresadas por los adolescentes, dentro de las que se encuentran, relaciones sexuales inesperadas, falta de dinero, no llevarlo consigo, la pareja no quiere, no se siente igual, le avergüenza conseguirlo o no había disponible, la falta información, entre otros (9). De acuerdo a dicho estudio, ninguna de las razones dadas por los jóvenes está asociadas a la estructura, la funcionalidad familiar ni al género, dando a entender que la protección es más del joven que de la influencia de su familia.

A pesar de lo anterior y de lo hallado en esta investigación, autores como Miller (15) afirman encontrar relación directa entre la comunicación (especialmente materna) y la promoción del uso del condón en la primera relación y que esto favorece la utilización del preservativo en las relaciones posteriores asegurando una vida sexual sana (15). Lavielle (9) encontró que la comunicación y la expresión del afecto en la familia como componentes de la funcionalidad familiar influyen de manera positiva en el adolescente al favorecer un nivel adecuado de autoestima y de calidad de vida. Afirma que "la comunicación en la familia acerca de la sexualidad y uso del condón, promueve el uso de este, tanto en la primera relación sexual como en las posteriores. Los adolescentes que perciben que la comunicación con su familia es abierta tienden a conductas de sexo seguro, entre ellas el inicio de la vida sexual a una edad no temprana, tener pocas parejas sexuales y el uso frecuente del condón" (9).

Este trabajo no evidenció diferencia entre hombre y mujer en cuanto al uso de algún método de planificación en la primera relación (tabla3). Respecto a lo que motiva a ambos géneros para usarlos, Rodríguez (16) se refiere a que la razón en cada caso es diferente porque "en las relaciones sexuales, el adolescente busca protegerse pensando en sí mismo y no en su pareja; cada uno asume el hecho de protección ante el riesgo que quiera evitar, en el caso del varón, una infección de transmisión sexual (ITS) y, en el de la mujer, el embarazo no deseado".

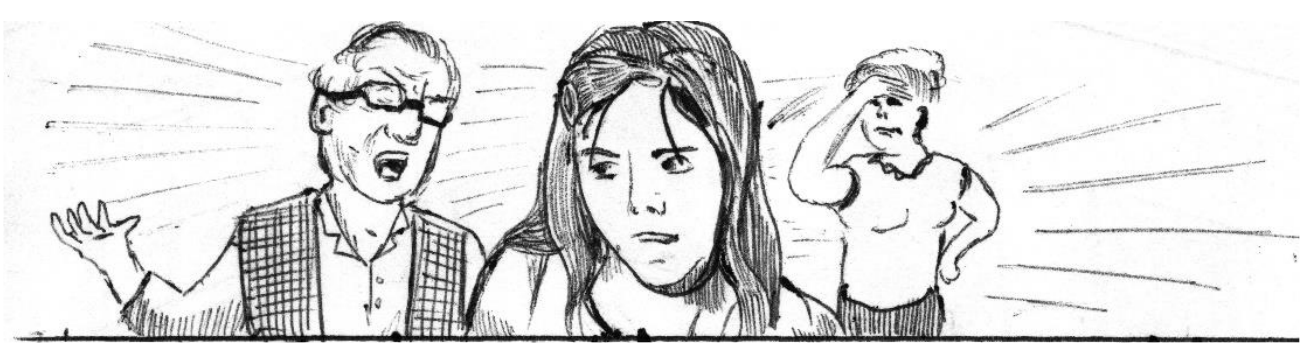

En conclusión, en cuanto a la pregunta de investigación, este estudio encontró una relación significativa de la funcionalidad familiar y el género con el inicio de vida sexual en adolescentes escolarizados; mientras que no se halló esta relación entre el uso de métodos anticonceptivos durante la primera relación sexual y la funcionalidad familiar o el género, aunque algunos autores (9) (15) afirman que la comunicación familiar es de gran importancia para educar a los jóvenes con respecto a este tema.

Como sea, la familia constituye el pilar de la sociedad pues es el centro de aprendizaje e inculcación de valores que le permiten al individuo salir a enfrentar la sociedad de manera integral y responsable (17); por tanto, es primordial que sea funcional, que el adolescente perciba en ella la posibilidad de participación, confianza y soporte emocional. Como lo confirma esta investigación, prima la función sobre la estructura, siendo la disfunción familiar un importante factor relacionado con los comportamientos sexuales de riesgo en torno a la sexualidad. Lo anterior se puede concluir, además porque las familias no nucleares pueden presentar ciclos vitales alternativos que le confieran un funcionamiento normal y es por esto que no se relacionan con dicho riesgo (11). 
Trabajos investigativos como el presente, permiten indagar sobre el debate que se dio en septiembre de 2016 en el que se vio involucrada la Procuraduría General de la Nación dadas las inquietudes presentadas por las acciones de prevención de conductas de riesgo en sexualidad en adolescentes (18), adelantadas por el DANE a través de la Encuesta de Comportamiento y Actitudes sobre Sexualidad en Niños, Niñas y Adolescentes Escolarizados (ECAS), que realiza la entidad a los menores de edad de las instituciones educativas del país. En dicho debate se expresó que las preguntas de la encuesta tienen un alto contenido sexual explícito e inapropiado para niños por lo que se corre el riesgo de despertar innecesariamente la curiosidad en los menores de edad y el posible desenlace de conversaciones, actuaciones y prácticas sexuales no aptas para todas las edades, "vulnerando el derecho de padres de familia a educar a sus hijos en temas tan trascendentales como la educación sexual". Ante esto, la Procuraduría exigió al DANE dar respuesta a inquietudes frente a posibles inconsistencias en la encuesta. La cuestión es si será deber de la Procuraduría emitir estas opiniones. Ahí dejamos la inquietud, sosteniendo que aún existe mucho por entender en torno la sexualidad de los adolescentes.

\section{AGRADECIMIENTOS}

Al doctor Juan Carlos González, docente de la cátedra de Investigación Social II y director del Departamento de Medicina Comunitaria de la Fundación Universitaria Juan N Corpas, quien nos brindó los conocimientos, herramientas y el optimismo necesarios para darle continuidad a tan importante proyecto de investigación para la comunidad. A las directivas de los colegios públicos de la localidad de Suba que accedieron a vincularse al estudio en pos de soluciones para los adolescentes de sus colegios. A la población adolescente participante.

\section{REFERENCIAS}

(1) Mendizábal J, Anzures L. La familia y el adolescente. Rev. Méd. Hospital General de México. 1999; 62(3): 191-197.

(2) Horwitz N, Florenzano R, Ringeling I. Familia y salud familiar. Un enfoque para la atención primaria. Boletín de la Oficina Sanitaria Panamericana. 2010; 98(2): 144-155.

(3) Gómez E, Castillo I, Cogollo Z. Predictores de disfunción familiar en adolescentes escolarizados. Revista Colombiana de Psiquiatría. 2013; 42(01): 72-80.

(4) Domínguez I. Influencia de la familia en la sexualidad adolescente. Revista Cubana de Obstetricia y Ginecología. 2011; 37(3): 387-98.

(5) Carvajal M, Essien J, Rey P, Bardales J. El adolescente, la familia y su educación sexual. Clin Invest Gin Obst. 2007; 34(6): 214-9.

(6) Atención integral a la primera infancia. Boletín 2. El aumento del embarazo en adolescentes en Colombia [Internet]. Bogotá D.C: Deceroasiempre.gov.co; 2013 [actualizada marzo de 2013; acceso 23 de octubre de 2016]. Disponible en:

http://www.deceroasiempre.gov.co/Prensa/CDocumentacionDocs/B olet $\%$ C3\%ADn $\% 20$ No. $\% 202 \% 20$ El\%20aumento\%20de $\% 20$ embara zos\%20adolescentes\%20en\%20Colombia.pdf

(7) Departamento Administrativo Nacional de Estadística (DANE). Boletín de prensa: Encuesta de comportamientos y actitudes sobre sexualidad en niñas, niños y adolescentes escolarizados. [Internet]. Bogotá. DANE.gov.co [actualizada 10 de mayo de 2013; acceso 23 de octubre de 2016]. Disponible en: https://www.dane.gov.co/files/investigaciones/boletines/ecas/bol_e cas 2012.pdf

(8) García E, Menéndez E, García P, Rico R. Influencia del sexo y del género en el comportamiento sexual de una población adolescente. Univ de Ovie Internet]. 2010 [citado 14 Octubre 2016]; 22(4): 606-612. Disponible: http://www.psicothema.com/pdf/3774.pdf

(9) Lavielle $P$, Jiménez F, Vásquez A, Aguirre M, Castillo M, Vega S. Impacto de la familia en las conductas sexuales de riesgo de los adolescentes. Rev Med Inst Mex Seguro Soc. 2014; 52(1): 38-43.

(10) González C. Conocimientos, actitudes y prácticas sobre sexualidad en una población adolescente escolar. Revista de Salud Pública. Universidad Nacional de Colombia. 2009; 11(1): 14-26.

(11) Salazar A, Santamaría A, Solano I, Lázaro K, Arrollo S, Araújo V et al. Conocimientos de sexualidad, inicio de relaciones sexuales y comunicación familiar, en adolescentes de instituciones educativas nacionales del distrito del Agustino, Lima (Perú). Revista oficial de la facultad de medicina humana de la Universidad de San Martín de Porres. 2007; 7(2): 79-85.

(12) Santander S, Zubarew T, Santelices LC, Argollo P, Cerda J, Bórquez $M$. Influencia de la familia como factor protector de conductas de riesgo en escolares chilenos. Revista médica de Chile. 2008; 136(1): 317-324.

(13) Pérez A, Pérez R, Martínez M, Leal F, Mesa I, Jiménez I. Estructura y funcionalidad de la familia durante la adolescencia: relación con el apoyo social, el consumo de tóxicos y el malestar psíquico. Atención Primaria. 2007; 39(2): 61-7.

(14) Márquez B, Pérez L, Sibaja B. Ser hombre, ser mujer en la sexualidad adolescente. Revista de Psicología de la Universidad de Chile. 2004; 13(2): 43-49.
(15) Miller K, Levin M, Whitaker D, Xu X. Patterns of Condom Use among Adolescents: The Impact of Mother-Adolescent Communication. American Journal of Public Health. 1998; 88(10): $1542-1544$.

(16) Rodríguez A, Álvarez L. Percepciones y comportamientos de riesgos en la vida sexual de los adolescentes. Revista cubana de Salud Publica [Internet]. 2006 [citado 15 de octubre de 2016]; 32(1). Disponible en: http://bvs.sld.cu/revistas/spu/vol32 1 06/spu08106.htmn

(17) Flórez E. Factores socioeconómicos y contextuales que determinan la actividad reproductiva de las adolescentes en Colombia. Revista Panamericana Salud Pública. 2005; 18 (6): 388-402.

(18) PNG. Procuraduría formula inquietudes al DANE frente al contenido de la encuesta que se realiza en las instituciones educativas del país. Boletín 754. Fecha Publicación: jueves, 22 septiembre 2016 01:49 PM. Disponible en:

http://www.procuraduria.gov.co/portal/Procuraduriaformula_inquietudes_al_DANE_frente_al_contenido_de_la_encuest a_que_se_realiza_en_las_instituciones_educativas_del_pa_s.news

(19) Cariote $E$. Influencia de los padres en la educación sexual de los adolescentes. Revista Médica. 2015; 1316-4910.

(20) Mendizábal J, Anzures L. La familia y el adolescente. Revista Médica del Hospital General de México. 1999; 62 (3): 191-197.

(21) Hoogan PD, Kitawua ME. The impact of social status, family structure, and neighborhood on the fertility of Black adolescents. Am J Soc. 1985; 90(4): 825-55.

(22) Mueller KE, Powers WG. Parent child sexual discussion: Perceived communicator style and subsequent behavior. Adolescence 2000 ; 25: $469-82$.

(23) González E, Molina T, Montero A, Martínez V. Factores familiares asociados al inicio sexual temprano en adolescentes consultantes en un centro de salud sexual y reproductiva en Santiago de Chile. Rev. Méd. Chile [Internet] 2013; 141(3): 313-319.Disponible en: http://dx.doi.org/10.4067/S0034-98872013000300005

(24) Ceballos GA, Campo A. Prevalencia del uso del condón en la primera relación sexual en adolescentes de Santa Marta, Colombia: Diferencia de género (Internet). 2005; 8(2). Disponible en: http://www.sidastudi.org/resources/inmagic-img/dd4276.pdf

(25) González E, Molina Gb, Montero A, Martínez N, Leyton C. Comportamientos sexuales y diferencias de género en adolescentes usuarios de un sistema público de salud universitarios. Revista Méd. Chile. 2007; 135: 1261-1269.

(26) López F, Carcedo R, Fernández N, Blázquez MI, Kilani A. Diferencias sexuales en la sexualidad adolescente: afectos y conductas. Publicaciones de la Universidad de Murcia. Anales de Psicología. 2011; 27(3): 791-799.

(27) Rangel $Y$, García $M$. Influencia del rol de género en la conducta sexual de riesgo en adolescentes universitarios. Index Enferm [Internet]. 2010 [citado el 14 de Octubre de 2016] 19(4): 245-248. Disponible en:

http://scielo.isciii.es/scielo.php?script=sci_arttext\&pid=S11321296201000330000

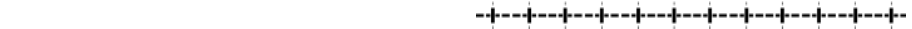

